\section{Open communication}

The TePe Communicator, which can be seen at stand E09, contains everything the dental professional may need to demonstrate to patients how to clean effectively, using the correct technique and TePe product.

It comes in a compact plastic case and includes a hand made lower dental arch with colour-coded interdental spaces, which clearly shows which TePe brush size should be used.

It also enables the patient to try products on the model before attempting them in their own mouths.

Samples of TePe products including the eight sizes of interdental brushes, Proximal, Interspace, Compact Tuft and TePe Select manual brushes are also included. A flip chart presenter with illustrations and patient leaflets complete the kit.

TePe Communicators are available to order exclusively from the Molar. Please

\section{Informative lectures}

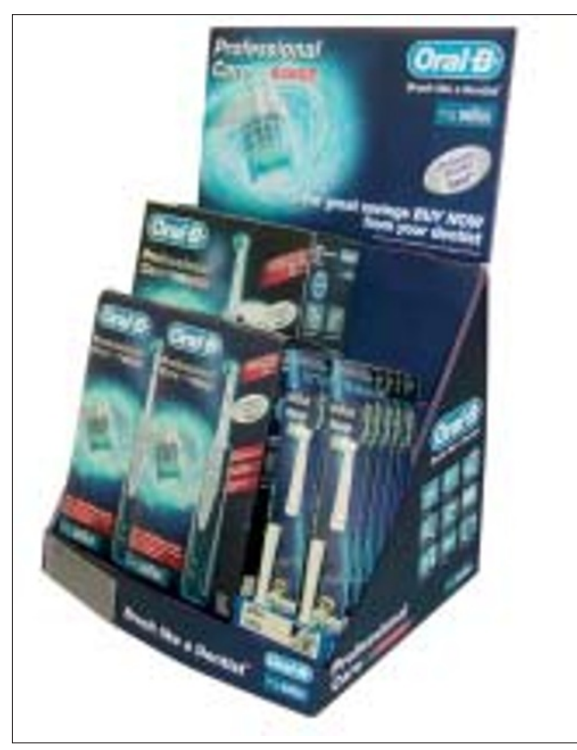

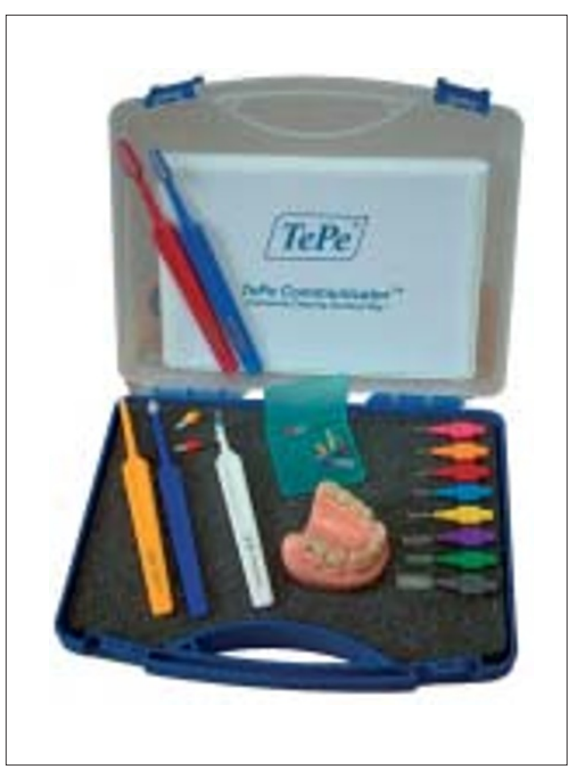

call 01934710022 for further information. Reader response number 59

At Showcase, Oral-B will be running a lecture area for their flagship product, The Oral-B Professional Care 7000.

The area is designed for visitors to learn, in an informal and relaxed environment, about the latest developments in power brushing.

According to Oral-B, recent surveys have found that power brushes with a rotating oscillating action have a superior cleaning technology and remove twice as much plaque as manual toothbrushes.

Oral-B will also be demonstrating other products at their stand at C06, among them specialist toothbrushes, CrossAction the Satin range of tape and floss, and children's toothbrushes and other sundry oral hygiene aids.

Reader response number 60

\title{
Making an impression at Dentsply
}

The Dentsply stand at E05-07 will feature Aquasil Ultra, a VPS impression material that can be adapted to oral fluid, thereby minimising the risk of retakes due to bubbles and voids in the impression.

Also available will be QuiXfil, a quick, durable, product which cures to $4 \mathrm{~mm}$ in 10 seconds and according to the company has a very low shrinkage rate of 1.7 per cent. The company will be exhibiting its full range of leading products from Flexichange, Cavitron, DenOptix, Refinement, X-Flow, Xeno, the established range of dental anaesthetics from Dentsply Pharmaceutical, to ProTaper, ProFile, Thermafil and also the comprehensive range of dental laboratory products. Reader response number $\mathbf{6 1}$

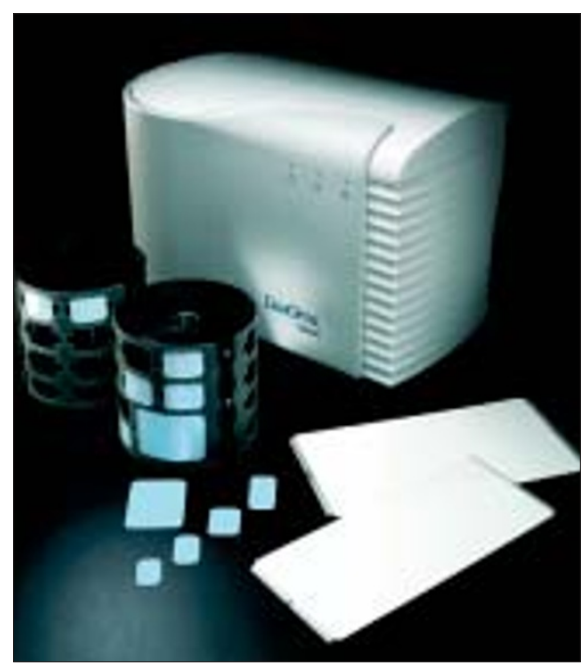

